\title{
Letter to the editor - serious asthma events with fluticasone plus salmeterol versus fluticasone alone
}

\author{
Surjit Singh* and Rimplejeet Kaur
}

AIIMS Jodhpur, Rajasthan, India

\section{To The Editor}

Stempel et al [1] compared serious asthma events in FluticasoneSalmeterol with Fluticasone alone in AUSTRI trial. Sum up of patients of four subgroups in severe asthma exacerbation (SAEx), 480 patients out of 5834 in fluticasone-salmeterol and 597 out of 5845 in fluticasone group had SAEx. The hazard ratio for severe asthma exacerbations in fluticasone-salmeterol group versus fluticasone alone is 0.78 (95\% CI, 0.69 to $0.89 ; \mathrm{P}=0.0002)$ as per ITT and $0.76(95 \% \mathrm{CI}, 0.67$ to 0.87 ; $\mathrm{P}=0.0002)$ as per PP analysis, although non-inferiority was establish with regard to composite safety endpoints ( $\mathrm{HR}=1.03$ (95\% confidence interval [CI], 0.64 to 1.66), Non-inferiority margin as 2). Stempel et al [1] states that there is no difference with regard to composite safety endpoints as per race. But if we compare the composite safety end point (hospitalization, endotracheal intubation, or death) or serious asthma related event (SARE) in black patients versus white patients in Salmeterol-fluticasone group, the hazard ratio is 2.41 (95\% CI, 1.13 to 5.14; $\mathrm{P}=0.02$ ), statistically significant. The hazard ratio for SARE in blacks' patients in fluticasone group is 1.80 (95\% CI, 0.80 to 4.04; P $=0.15$ ) versus white. The hazard ratio for SARE in black versus white patients in both treatment groups combined is 2.09 (95\% CI, 1.21 to 3.63; $\mathrm{P}=0.009$ ) (Figure 1). Thus, an additional finding of this study is that the risk of serious asthma related events is $109 \%$ higher among black patients as compared to white [2,3]. These finding are similar to the findings by The Asthma and Allergy foundation of America, which states that overall black to white asthma mortality ratio for the nation is 3.0 [2].

This may imply that we should be cautious while using combination of salmeterol-fluticasone while we can safely use fluticasone alone in blacks, as this study have established non-inferiority of both the therapies. However, meta-analysis is needed to further strengthen the evidence .

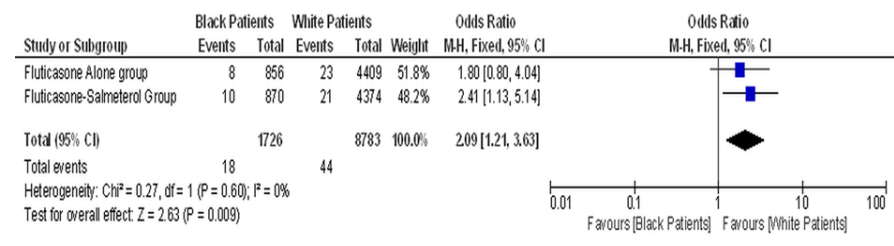

Figure 1. Forest plot of composite safety end points (serious asthma related events) in black versus white patients in both treatment groups

\section{Author's contribution}

Author SS, RK have written, edited and approved the final manuscript/correspondence.

\section{Declaration of interest}

Author SS, RK declare that they have no conflict of interest. Dr. Kaur has nothing to disclose. Dr. Singh has nothing to disclose.

\section{Role of funding source}

Not Applicable.

\section{Ethics committee / Institutional review board's permission}

Not Applicable.

\section{References}

1. Stempel DA, Raphiou IH, Kral KM, Yeakey AM, Emmett AH, et al. (2016) Serious Asthma Events with Fluticasone plus Salmeterol versus Fluticasone Alone. $N$ Engl J Med 374: 1822-1830. [Crossref]

2. Ethnic-Disparities-Burden-Treatment-Asthma-Report.pdf (2016). Available from: http://www.aafa.org/media/Ethnic-Disparities-Burden-Treatment-Asthma-Report.pdf

3. Nelson HS, Weiss ST, Bleecker ER, Yancey SW, Dorinsky PM. (2006) The Salmeterol Multicenter Asthma Research Trial: a comparison of usual pharmacotherapy for asthma or usual pharmacotherapy plus salmeterol. Chest 129: 15-26. [Crossref]
Copyright: (C2020 Singh S. This is an open-access article distributed under the terms of the Creative Commons Attribution License, which permits unrestricted use, distribution, and reproduction in any medium, provided the original author and source are credited.
${ }^{\star}$ Correspondence to: Surjit Singh, AIIMS Jodhpur, Rajasthan, India, Tel: 8003996895; E-mail: ss.sehmby@gmail.com, sehmby_ss@yahoo.com

Received: January 29, 2020; Accepted: February 10, 2020; Published: February 17,2020 